\title{
Kinematical properties of stellar populations in the Carina dSph galaxy
}

\author{
M. Monelli ${ }^{1}$, G. Bono ${ }^{2}$, M. Nonino ${ }^{3}$, P. Francois ${ }^{4}$, F. Thévenin ${ }^{5}$,

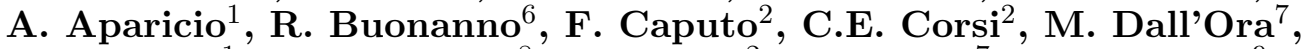 \\ C. Gallart ${ }^{1}$, A. Munteanu ${ }^{8}$, L. Pulone ${ }^{2}$, V. Ripepi ${ }^{7}$, H. A. Smith ${ }^{9}$, \\ P. B. Stetson ${ }^{10}$ and A. R. Walker ${ }^{11}$ \\ ${ }^{1}$ IAC-Instituto de Astrofísica de Canarias; ${ }^{2}$ INAF-Osservatorio Astronomico di Roma; \\ ${ }^{3}$ INAF-Osservatorio Astronomico di Trieste; ${ }^{4}$ Observatoire de Paris; ${ }^{5}$ Observatoire de la Côte \\ Azur; ${ }^{6}$ Dipartimento di Fisica, Universitá di Roma Tor Vergata; ${ }^{7}$ INAF-Osservatorio \\ Astronomico di Capodimonte; ${ }^{8}$ ICREA, University of Barcelona; ${ }^{9}$ Dept. of Physics, Michigan \\ State University; ${ }^{10}$ Dominion Astrophysical Observatory, Herzberg Institute of Astrophysics; \\ ${ }^{11}$ Cerro Tololo Inter-American Observatory;
}

\begin{abstract}
Multi-object spectrographs available on 8-m class telescopes provide the unique opportunity to directly investigate the kinematical and chemical properties of significant samples of resolved stars in galaxies of the Local Group. We present here the first results concerning an extensive study of stellar populations of the Carina dSph galaxy, based on data collected with the multi-object spectrographs FORS2 and FLAMES, both available at VLT. Preliminary estimates concerning the radial velocity distributions of the different samples are presented.
\end{abstract}

Keywords. techniques: spectroscopic; (galaxies:) Local Group; stars: kinematics; galaxies: individual (Carina dSph)

\section{Data reduction and results}

In the widely accepted $\Lambda$ CDM paradigm, dwarf galaxies play a major role in assembling giant galaxies. The resolved stellar populations of Local Group dwarfs offer the unique opportunity to study the processes governing their star formation history (SFH) and chemical evolution. The Carina dwarf galaxy is an interesting object, because of its complex SFH, its mix of stellar populations, its high Mass-to-Ligth ratio (Mateo 1998). However, despite of the three different bursts of star formation over a range of $\approx 10 \mathrm{Gyr}$, the photometric evidence supports a small spread in the chemical composition (Monelli et al. 2003). We present here the first results of a spectroscopic campaign devoted to study the kinematical and chemical properties of $\approx 1,450$ stars in Carina.

This work is based on two main data sets. Spectra of 1095 stars were downloaded from the ESO archive. These data have been collected with the multi-fiber FLAMES@VLT, in mid-resolution mode $(\approx 6,500)$ adopting the LR8 grating setup centered around the spectral region of the Calcium Triplet. Five fields were observed and, the targets only include bright Red Giant Branch (RGB), Asymptotic Giant Branch stars, plus field foreground stars of the Galaxy $(V<20.5)$. The total area covered is $\simeq 1 \mathrm{deg} \times 1 \mathrm{deg}$. The second data set consists of low resolution $(\mathrm{R} \approx 2,000)$ spectra collected with the multiobject FORS2@VLT, in different observing runs between February and December 2004. Five fields were observed, adopting the $1400 \mathrm{~V}$ grism with a wavelenght coverage ranging from 4560 to $5860 \AA$. The candidates were selected in order to have well-defined samples from the three main star formation events: young blue Main Sequence stars $(V>21)$, old Horizonthal Branch stars $(V \simeq 20.7)$, intermediate-age Red Clump stars $(V \simeq 20.5)$, and RGB stars $(V<20)$. 

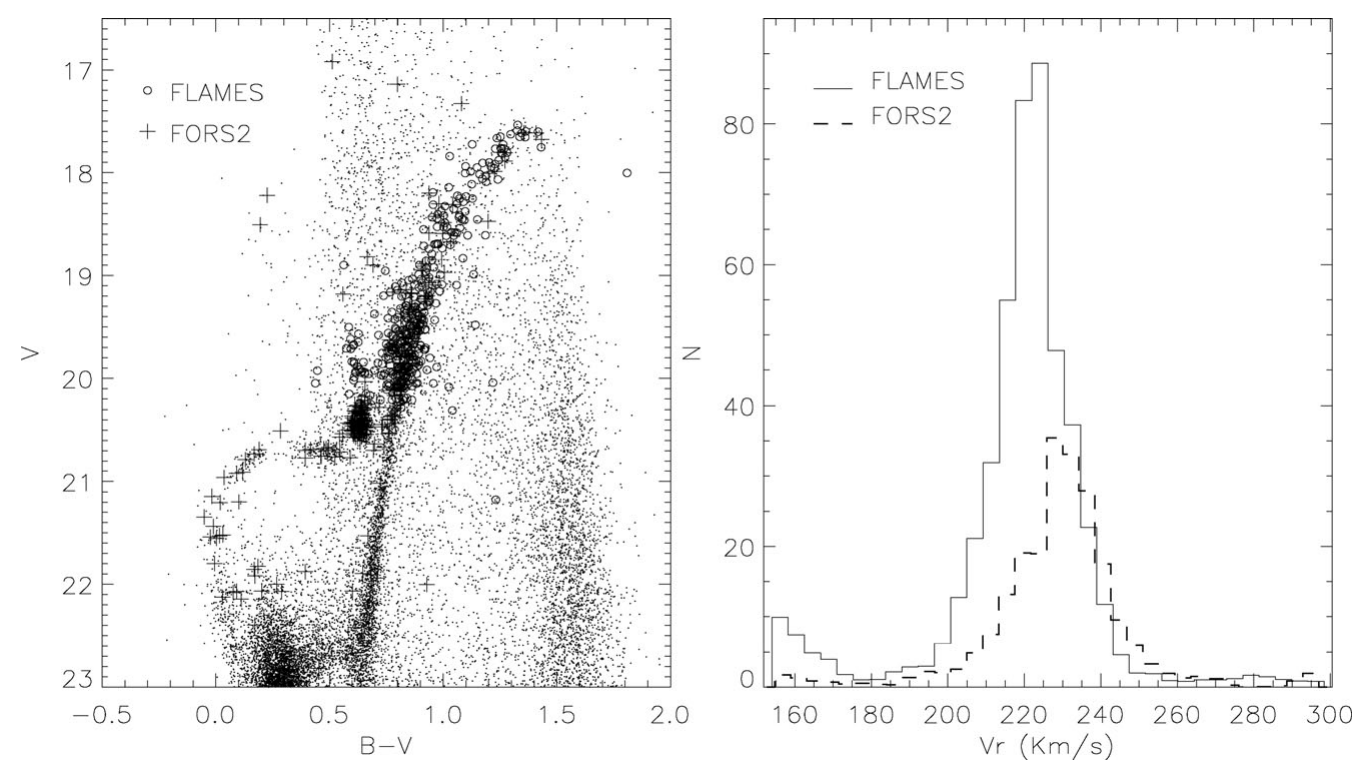

Figure 1. Left - CMD of Carina showinf the FLAMES (open circles) and FORS2 (crosses) candidates. Right - Histogram of the radial velocities comparing the two data sets.

The data reduction (bias, flat, extraction and wavelength calibration) was performed in the IRAF environment (Nonino et al. 2007). To avoid subtle systematics that could affect the radial velocity and chemical abundances analysis, we paid particular attention on the sky subraction (FLAMES) and on the detemination of the position of the star in the slit (FORS2). The left panel of Fig. 1 shows the Color-Magnitude diagram of Carina, with superimposed the FLAMES (open circles) and FORS2 (crosses) targets. The right panel shows the histogram of the radial velocity for the two samples. A preliminary analysis discloses that the main peak of the FLAMES sample $\left(\approx 221 \pm 5 \mathrm{~km} \mathrm{~s}^{-1}\right)$ is in good agreement with the values from the literature (Koch et al. 2006). Moreover, the peaks of the two distributions appear to be consistent, within the errors.

\section{Acknowledgements}

This work was partially supported by PRIN-INAF2005 (P.I.: A. Buzzoni), and by PRIN-INAF2004 (P.I.: M. Bellazzini).

\section{References}

Koch, A., Grebel, E. K., Wyse, R. F. G., Kleyna, J. T., Wilkinson, M. I., Harbeck, D. R., Gilmore, G. F., \& Evans, N. W. 2006, AJ, 131, 895

Nonino, M., Bono G. , Monelli M., et al. 2007, in: C. Sterken (ed.), The future of photometric, spectrophotometric and polarimetric calibration ASP Conference Series, Vol. 364 (San Francisco: ASP), in press

Mateo M., 1998, ARAA, 36, 435

Monelli M., Pulone, L., Corsi, C.E., Castellani, M., Bono, G., Walker, A.R., Brocato, E., Buonanno, R., Caputo, F., Castellani, V., Dall'Ora, M., Marconi, M., Nonino, M., Ripepi, V. \& Smith, H.A. 2003, AJ, 126, 218 